\title{
Rechnerintegration in kleinen und mittleren Unternehmen
}

\author{
Dr.-Ing. Helmut Bärthel, Prof. Dipl.-Ing. Karlheinz Kuchling, Dipl.-Ing. Günter Schubert
}

„Bei der Entwicklung von Informationssystemen, die eine durchgängige Unterstützung aller in einem Unternehmen zu bewältigenden Abläufe bieten können, ist nicht in erster Linie von den Leistungsmerkmalen konkreter EDV-Systeme auszugehen. Vielmehr sind hierbei die strategischen Unternehmensziele sowie die für die Erfüllung dieser Ziele erforderlichen Vorgangsketten zugrunde zu legen. Die EDV-Technik dient dann lediglich der Umsetzung der unternehmerischen Zielsetzung mit Hilfe konkreter Informationssyssteme und sollte die freie Gestaltung von Arbeitsabläufen und Organisationskonzeptèn ermöglichen."[1]

Die Suche nach sicheren Konzepten, angepaßt an individuelle Unternehmensstrukturen, ist an unterschiedlichen Forschungseinrichtungen ein Thema. An der Nahtstelle zwischen den Fachbereichen Betriebswirtschaft und Maschinenbau ist dies auch an der Technischen Fachhochschule Wildau ein Thema.

\section{Rechnerintegrierte Produktion als Begriff}

Integration ist ein viel verwendeter Begriff. Leider findet man in informationstechnischer wie auch in Ingenieur-Literatur recht wenig Beiträge, die diesen Begriff untersuchen. Die betriebswirtschaftlich orientierte Literatur bearbeitet dieses Problem häufig klarer.

\subsection{Integrierte Systeme, Integration}

Häufig ist zu erkennen, daß diese Begriffe sowohl zur Zustandsbeschreibung als auch zur Prozeßbeschreibung verwendet werden.

Eine etwas genauere Differenzierung wäre hier angebracht. Empfehlenswert ist sicher, im Zusammenhang mit Zustandsbeschreibungen von integrierten Systemen zu sprechen. Integrationsstufen sollten durch zusätzliche Attribute beschrieben werden.

Der Prozeß, der ein System in eine höhere Integrationsstufe überführt, sollte als Integration bezeichnet werden. Häufig verschmelzen dabei verschiedene Elemente, die anschließend nicht mehr isoliert betrachtet werden können. Auch ein Aufbau weiterer Beziehungen ist denkbar ([2], [3], [4]).

Analog zu solchen Begriffen muß die DV-Integration und integrierte Datenverarbeitung verstanden werden. Für eine integrierte Datenverarbeitung muß zu jedem Zeitpunkt gewährleistet sein, daß mit einem aktuellen Datenbestand gearbeitet wird.

Zusammen mit diesen diskutierten Begriffen erhalten auch Begriffe wie Datenintegration und Funktionsinte- gration entscheidende Bedeutung. Hierbei ist zu bemerken, daß eine Funktionsintegration eine Datenintegration voraussetzt (s. dazu auch [5]). Dabei soll die weit verbreitete Vorstellung beseitigt werden, daß Funktionsintegration eine zwangsläufige Folge von Datenintegration ist.

\subsection{Aspekte rechnerintegrierter Produktion in kleinen und mittleren Unternehmen}

Befaßt man sich mit Rechnerintegration im Unternehmen, kommt man am Begriff CIM nicht vorbei (s. dazu auch [6]).

So wie vom Ausschuß für wirtschaftliche Fertigung e. V. 1985 präsentiert, sind vom Begriff CIM alle technischorganisatorischen Bereiche erfaßt (Bild 1 nach [7, S. 39]).

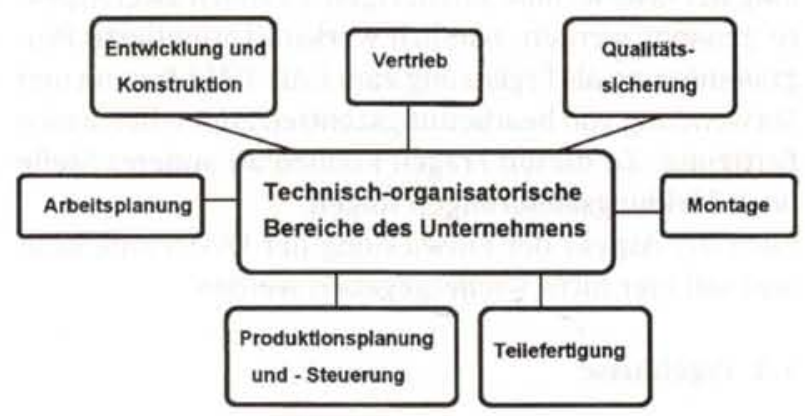

Bild 1: Technisch-organisatorische Unternehmensbereiche nach |7|

Widmen wir uns nicht dem CIM-Begriff, auch nicht den CIM-Ruinen, sondern untersuchen wir einige ausgewählte Aspekte der rechnerintegrierten Produktion [8].

\subsubsection{Die Organisation}

In jedem Unternehmen haben Fertigungstechnologie, Produktionsspektrum, Auftragslage und Größe des Unternehmens Einfluß auf die Aufbau- und Ablauforganisation (siehe auch [9]).

Im Zusammenhang mit der Rechnerintegration tauchen natürlich neue Aspekte in der Unternehmensorganisation auf mit entsprechenden funktionellen Zuordnungen. Hier sei beispielhaft der Systembetreuer genannt. In kleinen Unternehmen ist es die Kombination aus dem Fachmann und den DV-Kenntnissen bzw. Systemkenntnissen. Aufgrund der dünnen Personaldecke des kleinen Unternehmens ist es eine Schlüsselposition. Absatzlage und Geschwindigkeit sowohl des Informationsflusses als auch des Materialflusses bestimmen hochgradig die Effizienz der Ablauforganisation des Unternehmens.

Für das kleinere Unternehmen wird jedoch immer die Unterstützung bei der Funktionserfuillung im Vorder- 
grund stehen. Man kann sagen, daß sich die Organisationstheorie sicher noch länger mit einer angemessenen Mensch-Rechner-Funktionsverteilung auseinandersetzen muß.

\subsubsection{Der Mensch}

Im kleinen Unternehmen, eigentlich in jedem Unternehmen, muß das kreative Potential der Mitarbeiter voll zur Geltung kommen, d. h. der Handlungsspielraum der Mitarbeiter ist in Grenzen zu erweitern. Damit müssen dem Mitarbeiter die Zusammenhänge seiner Tätigkeit besser bewußt gemacht werden. Dieser Punkt ist ganz sicher streitbar.

Zudem läßt sich daraus ein ganz erhebliches Qualifikationsproblem ableiten. Der Fachmann muß zusätzlich zu seinen Fachkenntnissen und Erfahrungen Kenntnisse auf dem Gebiet der Informationstechnologie erwerben. Eine Reihe von Geschäftsführern unterschätzen dieses Problem gewaltig. Das Thema Schulung ist fuir so manches Unternehmen gar kein Thema oder nur ein Thema am Rande des Geschehens.

\subsubsection{Die Technik}

Im Zusammenhang mit der Rechnerintegration im Unternehmen sind hier sicher sowohl die Fertigungstechnik als auch die DV-Technik zu nennen.

Inhalt dieser Gedanken soll es nicht sein, die Entwicklung der CNC-Technik aufzuzeigen. Es sollen zwei Aspekte genannt werden, nämlich werkstattorientierte Programmierung als Ergänzung zum CAD-CAM-System und Verwendung von Bearbeitungszentren zur bedienarmen Fertigung. Zu diesen Fragen können an anderer Stelle noch Meinungsäußerungen folgen.

Auch der Aspekt der Entwicklung der DV-Technik kann und soll hier nicht wiedergegeben werden.

\subsection{Ergebnisse}

Aufgrund einer Reihe von Untersuchungen in unterschiedlichen Unternehmen konnte festgestellt werden, daß die Bandbreite der Rechnerintegration sehr breit ist. Es ist der Stand „0“ zu finden bis hin zum „Steuerungs-, Betriebssystem- und Netzsalat". Man kann durchaus von Opfern der Rechnerintegration sprechen.

Eine der häufigsten Ursachen für letztgenanntes Ergebnis ist der DV-Wildwuchs im Unternehmen. Oft ist bei Geschäftsführern fehlendes Einfuihlungsvermögen für die Probleme der Rechnerintegration zu beobachten. Es wird erwartet, daß sich die hohen Investitionen schnell amortisieren.

Mangelhafte, d. h. unvollständige, unverständliche und mit zu wenig Übungszeiten versehene Schulungskonzepte für die Unternehmen sind anzutreffen.

Erfolgreiche Lösungen sind nur durch partnerschaftliche Kooperation zwischen Anbieter und Anwender zu erreichen. Hochschulen können dabei sehr gute Mittler oder Partner sein. Die Technische Fachhochschule Wildau ist auf Grund ihres Wissens- und Erfahrungspotentials ein Partner, der sowohl die Systemauswahl auf der Grundlage der beim Klein- oder mittleren Unternehmen vorliegenden Gegebenheiten vorbereiten kann als auch die Realisierung begleiten kann.

\section{CAD-CAM-System für einen Formenbauer} ([10]-[12])

\subsection{Einleitung}

Die Technische Fachhochschule Wildau erbringt gemeinsam mit dem Technologietransfer- und Weiterbildungszentrum an der TFH ein umfangreiches Leistungsangebot für die Industrie, im besonderen für kleine und mittlere Unternehmen. Es sind dies sowohl Studienarbeiten, praktische Studiensemester und Diplomarbeiten der Studenten und Forschungsleistungen der Fachbereiche wie auch komplexe Beratungsleistungen in Form von Analysen, Studien und Projekten. Der Doppeleffekt aus hauseigener Forschung und Erprobung sowie kostengünstige Leistung für die Industrie führt zu verstärkter Zusammenarbeit zwischen der Technischen Fachhochschule und kleinen und mittleren Unternehmen verschiedenster Industriezweige.

Im vorliegenden Fall ist eine Machbarkeitsstudie für einen mittelständischen Formenbauer im Land Brandenburg (zukünftig als auftraggebendes Unternehmen AU benannt) anzufertigen. Es werden ausgewählte inhaltliche Schwerpunkte vorgestellt.

\subsection{Statement}

„Nur durch die Konstruktion und Fertigung hochkomplizierter Formteile werden wir als Unternehmen unsere Marktkompetenz behaupten und ausbauen. Der Markt für einfache Formen ist $\mathrm{z}$. T. von deutschen Herstellern, vorrangig aber von Firmen der ehemaligen RGW-Staaten, abgedeckt. Unsere Kunden kommen zu 85\% aus dem Automobilbau. Wer hier mitarbeiten will, muß fachlich kompetent sein, termingemäß und qualitätsgerecht liefern - zu einem guten Preis. D. h. unsere bisher sehr handwerklich geprägte Fertigung muß technisch und organisatorisch aufgerüistet werden."

Mit diesen Worten des technischen Geschäftsführers des zu beratenden Unternehmens war die zu lösende Aufgabe grob umrissen: Untersuchungen zum Einsatz eines durchgängigen 3D CAD-CAM-Systems von der Konstruktion bis zur Fertigung.

\subsection{Aufgaben- und Zielstellung}

Die als durchgängige 3D CAD-CAM-Lösung benannte Lösung fuir das AU hat vorrangig die folgenden Aufgaben und Ziele zu integrieren.

\subsubsection{Aufgabenstellung}

- Analyse des vorhandenen konstruktiven und fertigungstechnischen Prozesses nach konstruktiven, technologischen und betriebswirtschaftlichen Kriterien

- Erarbeitung der Schwachstellenanalyse und Klassifizierung der Veränderungspotentiale

- Analyse moderner CAD-Arbeitstechniken in Verbindung mit CAM-Lösungen und Bewertung der angebotenen Hard- und Softwarelösungen

- Erarbeitung einer Konzeption für einen modernen fertigungstechnischen und organisatorischen Arbeitsablauf 
- Konzeption für ein modernes Werkstattlayout mit Einbindung aller aus der Fertigungsumgebung ableitbaren Einflußfaktoren

- Ableitung ergonomischer Bedingungen zur Gestaltung der CAD-CAM-Arbeitsplätze und konventionellen Arbeitsplätze

- Konzeption zur meßtechnischen Lösung für die Kontrolle der konstruierten und gefertigten Formen mit Erfassung der Meßergebnisse

- Konzeption zur Einbindung der datenverarbeitenden Bedingungen und Ausrüstungen in den Konstruktions- und Fertigungsprozeß einschl. der Präzisierung des vorhandenen Werkstattlayouts

- Konzeptionelle Gestaltung des Qualifizierungsprogrammes auf der Grundlage der neuen CAD/CAMTechnik für die Beschäftigten

\subsubsection{Zielstellung}

- Kommisionsbezogene Auftragsbearbeitung (Annahme, Verfolgung, Kalkulation, Auslieferung, Auswertung)

- Konstruktion (3D-Modellierung, Farbschattierung, Flächen- und Volumenanalyse) von Formen und Werkzeugen mittels CAD-System unter besonderer Beachtung der Losgröße 1

- Übernahme von 3D-Erzeugniskonstruktionen der Kunden und eines im Auftrag des AU arbeitenden Konstruktionsbüros in das hauseigene CAD-CAD-System

- Umwandlung der CLDATA-Files zu NC-Programmen der entsprechenden Steuerung

- Erstellung von Meßprogrammen

- Erstellung von Erstmusterprüfberichten

- Einbindung vorhandener Systeme und Komponenten aus dem maschinentechnischen Bereich

\subsection{Bearbeitungsablauf}

Für den Bearbeitungsablauf der Machbarkeitsstudie wurden 7 Schritte definiert:

- Schritt 1: Aufgabenstellung

- Schritt 2: IST-Zustand mit kritischer Würdigung

- Schritt 3: SOLL-Zustand für eine CAD-CAM-Lösung

- Schritt 4: Diskussion des SOLL-Zustandes

- Schritt 5: SOLL-Konzeption 95'

- Schritt 6: Ablaufplan

- Schritt 7: Erarbeitung der Fördermittelanträge Es sollen nun ausgewählte Abschnitte, vorrangig aus den Schritten 2, 3 und 5, vorgestellt und diskutiert werden.

\section{Zu Schritt 2: IST-Zustand}

\section{Aufgaben/Produkte}

- Aufträge für Werkzeuge und Formen kommen von festen Kunden.

- Ein sehr unterschiedliches Produktionssortiment liegt nach Abmessungen, Masse und geometrischer Form vor (max. Abmessungen $1000 \times 1200 \mathrm{~mm}$ ).

- Die Losgröße beträgt 1.

- Grundlage eines Auftrages ist entweder eine Erzeugniszeichnung (der Formenbauer erbringt dazu Konstruktion und Fertigung) oder eine Konstruktionszeichnung aus einem Fremdkonstruktionsbüro.
- Die Kunden kommen überwiegend aus den Bereichen Motoren-, Automobil- und elektrotechnische Industrie, Hausgerätetechnik, Möbelindustrie und Fensterproduktion.

- Eine Quantifizierung der Aufträge (Aufträge/Zeiteinheit) ist wenig sinnvoll, da der Zeitaufwand zur Bearbeitung eines Auftrages extrem schwankt. So umfaßt eine Werkzeugkonstruktion im Mittel 4 bis 5 Zeichnungen DIN A0 plus Elektrodenkonstruktion mit ca. 70 Zeichnungen DIN A4/A3 für einen Auftrag.

- Die Aufgabenbreite und -tiefe stellt an die Beschäftigten hohe Ansprüche, die auf Grund der derzeit vorhandenen Rechentechnik, der wenigen Ausbildung/ Schulung sowie der vorhandenen Werkzeugmaschinen nach Art und Zustand einschl. ihrer Steuerungsvielfalt nur teilweise erfüllt werden können.

- Es ergeben sich daraus u. a. extreme Zeitaufwendungen für Spannarbeiten. Die Arbeitsvorbereitung arbeitet mit bescheidenster Rechentechnik, dies führt bis hin zu Verfahrensauswahlproblemen, d. h. die vorhandene Technik wird unabhängig von einer Verfahrensoptimierung eingesetzt.

- Eine Qualitätssicherung der fertigen Form kann nicht gegeben werden, da sowohl Meßtechnik als auch Ausbildung fehlen.

- Eine Konstruktionskontrolle findet nicht statt.

- Bei Konstruktionen im eigenen Hause werden Varianten auf der Grundlage von Mitarbeitererfahrungen diskutiert.

- Es gibt kein firmeneigenes Zeichnungsnummersystem, derzeit wird nach Kommissions-Nr. geordnet.

- Eine kommissionsbezogene Nachkalkulation findet nicht statt.

- Durch die Werker werden während der Bearbeitung Änderungen am Bearbeitungsablauf vorgenommen, aber nicht gespeichert. Der Änderungsrüicklauf zur Konstruktion und die Einarbeitung dieser Änderungen wären rein zufällig.

- Auf Grund von Kundenforderungen werden beinahe alle Spritzgußformen geändert. Dies ist nicht als direkter Mangel zu werten, da durch die Herstellung des ersten Werkstïckes notwendige Änderungen an der Form sichtbar werden.

\section{Personal}

Die 17 Beschäftigten gliedern sich zunächst beinahe klassisch in die Geschäftsleitung mit Sekretariat, die Arbeitsvorbereitung und Konstruktion sowie die Arbeitsvorbereitung mit Fertigung. Doch schon dabei zeigt sich:

- Die Tätigkeiten der Arbeitsvorbereitung werden sowohl von den Konstrukteuren als auch von den Werkern ausgefuihrt und

- 12 Werker unterstehen dem Technischen Leiter, der gleichzeitig konstruktiv und in der Arbeitsvorbereitung tätig ist.

Qualifikationsseitig sind für die gegenwärtige Produktionsdurchführung alle erforderlichen Voraussetzungen vorhanden.

\section{Maschinentechnik}

Die vorhandenen Maschinen zum Fräsen und Drahterodieren sind zwischen 5 und 10 Jahren alt und in sehr 
unterschiedlichem Zustand, woraus sich sehr früh notwendige Überlegungen für den Einsatz eines CAD-CAMSystems ergeben.

Eine Meßmaschine, eine Werkzeugmaschine zum Messen bzw. Kapazität zum Messen auf einer Werkzeugmaschine sind nicht vorhanden, ein Werkzeugvoreinstellgerät ist nicht im Einsatz.

\section{Steuerungslösung}

Die vorhandene Hard- und Softwaretechnik einschl. Peripherie entspricht dem Baujahr 1988, der Zustand ist befriedigend.

Im Rahmen der kritischen Würdigung des IST-Zustandes werden als wesentliche Komponenten herausgearbeitet:

- Ausgehend von der Aufgaben- und Zielstellung zu vorliegender Aufgabe ist durch die Analyse erkennbar, daß die beabsichtigte CAD-CAM-Lösung nur der erste Teil (vorbereitende und steuernde Komponenten) ist einer zweiteiligen Vorgehensweise zur

- Stabilisierung der Arbeitsplätze,

- termin- und qualitätsgerechten Produktion und Auslieferung,

- flexiblen Reaktion auf unterschiedliche Aufträge.

- Die Analyse zeigt, daß der zweite Teil, die eigentliche Herstellung, nicht dieser o. g. Zielstellung entspricht. Der Zustand der Werkzeugmaschinen einschließlich der zugehörigen Informationsverarbeitung bietet eine divergierende Bandbreite und beinhaltet Gïtegradeunterschiede, die eine kontinuierliche und qualitätsgerechte Fertigung mit hoher Flexibilität nicht zulassen.

Das gesamte Werkzeug-, Meßzeug- und Spannzeugwesen ist weitestgehend Improvisation.

- Hinzu kommt die Beachtung und Veränderung von derzeitigen „Randproblemen“ wie das Klima und die Lichtverhältnisse in der Fertigungshalle sowie die staubarme Maschinenreinigung, aber auch die Einordnung von Meßtechnik und Rechentechnik im fertigungsnahen Bereich.
Zusammenfassend ist erkennbar: Im Sinne einer Präzisierung der Aufgabenstellung ist aus der Analyse im Unternehmen und auf der Grundlage der beim Auftragnehmer vorliegenden Erfahrungen von einer weiteren Doppelfunktion für die Lösung auszugehen. Es handelt sich dabei zum einen um die notwendige annähernde Niveaugleichheit von Ausrüstungen zur Vorbereitung und zur Durchführung der Produktion („durchgängige Niveaugleichheit") und zum anderen um die Beachtung eines stufenweisen Aufbaues sowohl der vorbereitenden Funktionsträger (Rechentechnik für die Konstruktion, das Entwerfen, die Kalkulation, die Angebotserarbeitung, ...) als auch von Ausrüstungen der Fertigung, des Messens usw.

\section{Zu den Schritten 3-5: SOLL-Zustand-Diskussion - SOLL-Konzeption}

Im Schritt 3 SOLL-Zustand wurde das entsprechend der Aufgabe in Frage kommende Lösungsfeld für die durchgängige CAD̉-CAM-Lösung herausgearbeitet. Umfangreiche Erfahrungen unserer Fachhochschule sowie Informationen von Herstellern und Lieferern waren die Voraussetzungen dafür, diesen arbeitsaufwendigen Schritt in einer verhältnismäßig kurzen Zeit dem AU vorzulegen. Beispielhaft enthält die folgende Tabelle 1 eine Variante aus der Variantenmenge der Hardware für die Gerätekonfiguration 1:

\begin{tabular}{|c|c|c|c|}
\hline $\begin{array}{l}\text { Kenn- } \\
\text { Nr. }\end{array}$ & Hardware & Anwendung & $\begin{array}{l}\text { Hersteller/ } \\
\text { Anbieter }\end{array}$ \\
\hline 1 & $\begin{array}{l}\text { Workstation HP9000/715-75: } \\
715-75 \text {. HCRX.24Z20" Farbmonitor } \\
128 \text { MB RAM, I GB Festplatte } \\
2 \text { GB SCSI-DAT-Laufwerk inkI. } \\
\text { UNIX-Betriebssystem } \\
\text { CD-ROM-System, SCSI für HP9000 }\end{array}$ & $\begin{array}{l}\text { 3D CAD/CAM-Arbeits- } \\
\text { platz für } \\
\text { HP/PE SolidDesigner }\end{array}$ & $\begin{array}{l}\text { Hewlett-Packard } \\
\text { INC }\end{array}$ \\
\hline 1.1 & $\begin{array}{l}\text { Plotter HP-Designjet } 600 \wedge 0 \\
\text { Jet-Direct-Netzwerkkarte } \\
\text { Speichererweiterung } 8 \mathrm{MB} \\
\text { Supportpack (3].-Hardware-Service) }\end{array}$ & $\begin{array}{l}\text { Ausgabe von Zeich- } \\
\text { nungen } \mathrm{A} 3 \text { bis } \mathrm{A} 0\end{array}$ & \\
\hline
\end{tabular}

Tabelle 1: Hardware Variante 3 INC für die Gerätekonfiguration 1 in Bild 2

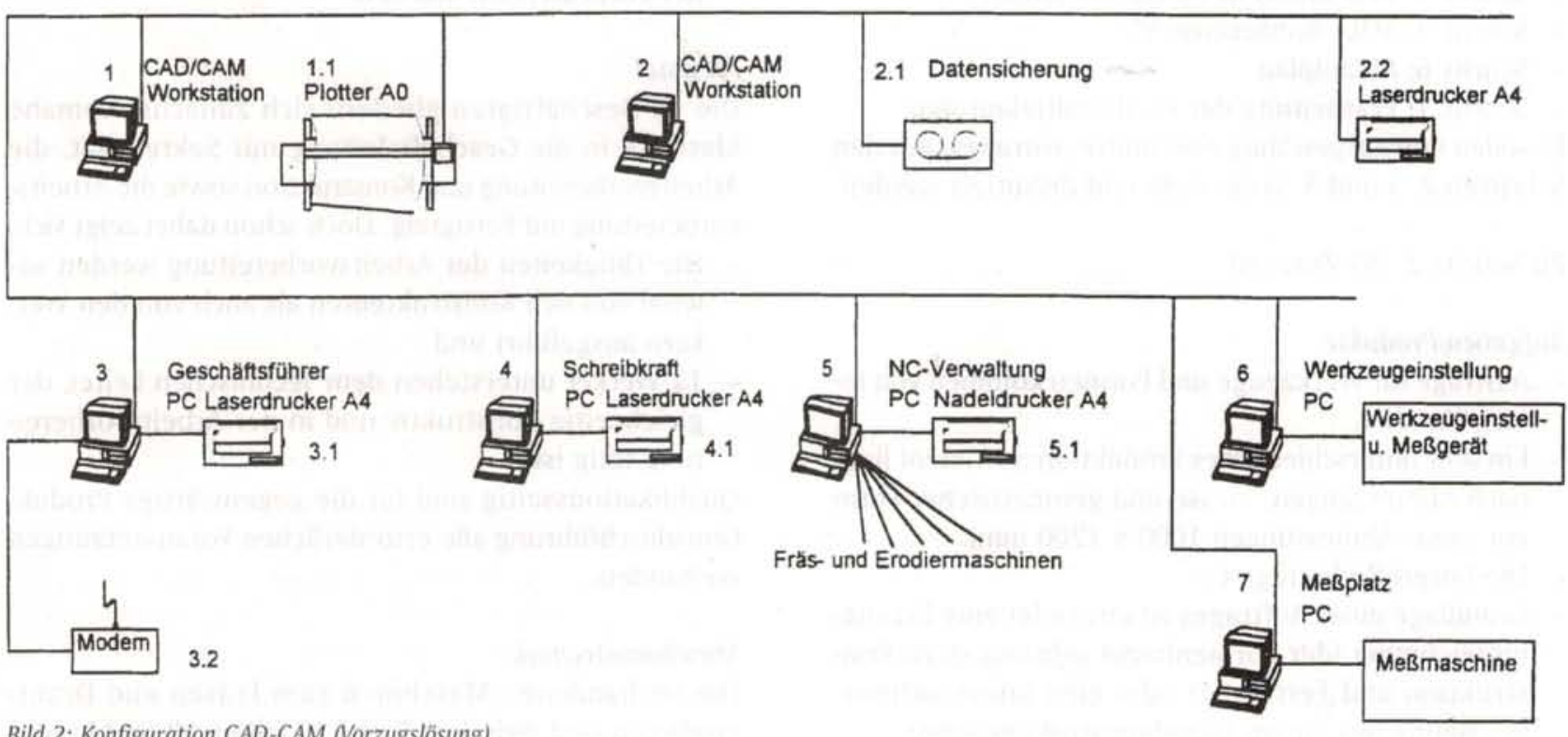


Beispielhaft aus der Menge der möglichen Lösungen ein Element einer Software Variante:

\begin{tabular}{|l|l|}
\hline Software & Anwendung \\
\hline HP- UX 9.05 & $\begin{array}{l}\text { Betriebssystem } \\
\text { (gehört zum Lieferumfang der Hardware) }\end{array}$ \\
\hline CAMAX & $\begin{array}{l}\text { 3D-Konstruktion 2D Zeichnungserstellung } \\
\text { 2D/3D CAM-Software } \\
\text { alternativer Zugriff tiber NFS von 2. WS aus }\end{array}$ \\
\hline $\begin{array}{l}\text { ME 10 bestehend aus: } \\
\text { Basissystem HiddenLine und DXF } \\
\text { Datenträger } \\
\text { Handbücher }\end{array}$ & 2D-Konstruktion von Formwerkzeugen \\
\hline $\begin{array}{l}\text { ASCAM ATLAS } \\
\text { ZVS. Erstlizenz }\end{array}$ & $\begin{array}{l}\text { 3D-Konstruktion } \\
\text { Fàchenrückführung } \\
\text { alternativer Zugriff tiber NFS von 2. WS aus }\end{array}$ \\
\hline SURFACER Komplettpaket & $\begin{array}{l}\text { Grafische Benutzeroberfliche fir MS.Windows- } \\
\text { Programme }\end{array}$ \\
\hline
\end{tabular}

Tabelle 2: Software Variante 2 ASCAM für die Gerätekonfiguration 1

Die Hard- und Softwaretechnik wurde in drei Varianten vorgestellt. Alle Varianten wurden durch Angebote gestiutzt, so daß auch Preise und Lieferbedingungen vorlagen.

Ergänzend zu diesen Aussagen erfolgten Betrachtungen zu möglichen und notwendigen Veränderungen der Aufgaben- und Personalstruktur. Das nachfolgende Bild 3 zeigt die neue Personalstruktur als Organigramm. Charakteristisch sind die saubere Trennung vorbereitender und ausführender Tätigkeiten, die Kopplung von Konstruktion und Arbeitsvorbereitung sowie die zentrale Position der Arbeitsvorbereitung als Instrument der Geschäftsleitung.

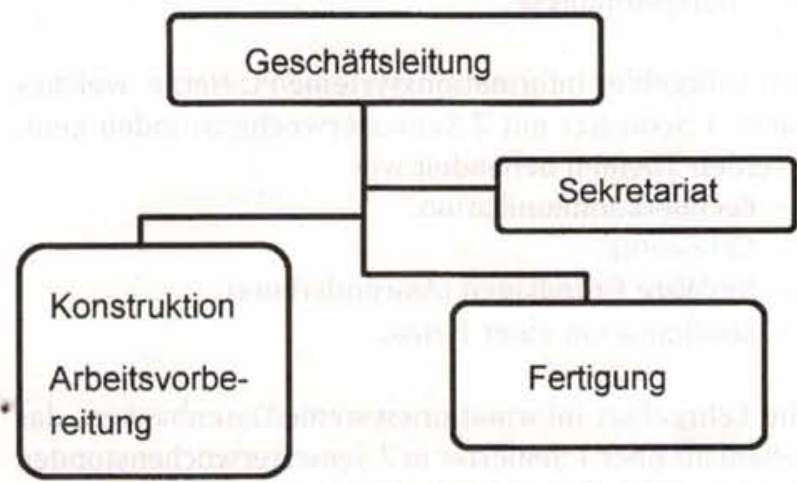

Bild 3: Organigramm der zukünftigen Personalstruktur

Wesentlich erschien für den Übergang zu einer Fertigung mit integriertem CAD-CAM-System die eindeutige Aufgabendarstellung der Arbeitsvorbereitung. Die folgende Tabelle 3 enthält die möglichen Aufgabengebiete der AV und untersetzt sie mit den wesentlichen Aufgaben. Im Rahmen der vorliegenden Machbarkeitstudie wurde auch die Thematik Flächen und Räume abgearbeitet. Schwerpunkte waren die veränderten räumlichen Bedingungen in der Konstruktion; ebenfalls ein Zielpunkt war die Werkstatt, u. a. mit den Fragen der räumlichen $\mathrm{Zu}$ ordnung der Meßeinheit, des Werkzeugvoreinstellgerätes und der mögli chen Kabelwege. Detaillierte Aussagen dazu erfolgen zum Zeitpunkt der Planung nach Auswahl und Bestätigung der Vorzugsvariante.

\begin{tabular}{|c|c|c|}
\hline \multicolumn{3}{|c|}{ Arbeitsvorbereitung } \\
\hline Rationalisierung & Arbeitsplanung & Arbeitssteuerung \\
\hline $\begin{array}{l}\text { - Mechanisierung und } \\
\text { Automatisierung }\end{array}$ & $\begin{array}{l}\text { - Koordinierung von } \\
\text { Aufgaben }\end{array}$ & - Auftragsdisposition \\
\hline & - Fertigungsvorbereitung & - Auftragsvorbereitung \\
\hline - Vereinheitlichung & - Fertigungsplanung & - Materialdispoisiton \\
\hline $\begin{array}{l}\text { - Erkennen von Schwach. } \\
\text { stellen }\end{array}$ & - Öberwachungsaufgaben & - Terminplanung \\
\hline & - Arbeitsplanerarbeitung & $\begin{array}{l}\text { - Bereitstellung von Mate- } \\
\text { rial und Information }\end{array}$ \\
\hline Wirtschaftlichkeitsrechnung & - Bedarfsplanung & \\
\hline - Optimale Kenngrößen & - Betriebsmittelplanung & \\
\hline - Beschäftigungsgrad & $\begin{array}{l}\text { - WZ- und Vorrichtungs- } \\
\text { konstruktion }\end{array}$ & \\
\hline - Verfahrensvergleich & - Fertigungseinrichtungen & \\
\hline $\begin{array}{l}\text { - Vergleichsrechnung. } \\
\text { Kostenrechnung }\end{array}$ & - Instandhaltungsplanung & \\
\hline - Kalkulation & - Werkstättenplanung & \\
\hline
\end{tabular}

Tabelle 3: Inhalt der zukünftigen Arbeitsvorbereitung

Der 4. Schritt, Diskussion des SOLL-Zustandes, konnte durch die aktive Einbeziehung des Managements und der Mitarbeiter des AU, die später mit dem System arbeiten werden, konstruktiv und zügig abgearbeitet werden. Auf der Grundlage der in der Machbarkeitsstudie vorgestellten Varianten, einer Präsentation des Lösungsfeldes durch die TFH Wildau (AN) sowie Präsentationen von Systemanbietern entschied sich das AU für die Variante 1 Pro/ ENGINEER. Charakteristisch für dieses System sind u. a.:

- Pro/ENGINEER ist ein elementorientiertes CAD-CAMSystem,

- Pro/ENGINEER basiert auf einem 3D-Volumenmodell,

- Pro/ENGINEER ist voll parametrisiert,

- Pro/ENGINEER arbeitet mit einer einheitlichen Benutzeroberfläche aller Module,

- Pro/ENGINEER besitzt standardisierte Schnittstellen und erfüllt höchste Anforderungen im Freiformflächenbereich und der Variantenkonstruktion,

- Pro/ENGINEER besitzt weitere wichtige Eigenschaften bis hin zur möglichen Produktivitätssteigerung in Konstruktion und Fertigung um den Faktor 3.

Die SOLL-Konzeption als 5. Schritt der Studie ist charakterisiert durch die CAD-CAM-Konfiguration, wie sie in Bild 2 dargestellt ist. Für die in der Konfiguration enthaltenen Kenn-Nr. wurden die Anforderungen und die zugehörige Hardware mit Bezugsquellen erarbeitet. Die folgende Tabelle 4 enthält Anforderungen und Hardware. Bezugsquellen und Angebotsunterlagen sowie die Software-Zuordnung sind Inhalt der Vorzugslösung und liegen beim Autor vor.

Ein weiterer Schwerpunkt der SOLL-Konzeption ist der Ablaufplan für die Planungs- und Realisierungsphase, dies um so mehr, da auch Planungsaktivitäten und eine begleitende Realisierung durch die TFH Wildau angedacht sind. Der Ablaufplan enthält etwa 30 Aktivitäten, von der Lieferung, Montage und Inbetriebnahme des CAD-CAM-Systems über Aktivitäten wie Systemschulungen in Grund- und Aufbaukursen, Übungseinheiten bis hin zur Erbringung von Bauleistungen für die Verkabelung und zum Aufstellen und Abschirmen der Meßeinheit sowie weitere Aktivitäten einschl. der gegenwärtig bekannten oder eingeschätzten Zeitbedarfsanteile. 


\begin{tabular}{|c|c|c|}
\hline $\begin{array}{c}\text { Kenn-Nr } \\
\operatorname{ans} \text { Kantes }\end{array}$ & Anforderungen & Hardware \\
\hline \multirow[t]{3}{*}{1} & $\begin{array}{l}\text { 3-D CAD/CAM-Arbeitsplatz für } \\
\text { Pro/Engineer }\end{array}$ & $\begin{array}{l}\text { Workstation HP9000/715-75, CRX } \\
32 \text { MB RAM, } 525 \text { MB Festplatte inkI. } \\
\text { UNIX-Betriebssystem }\end{array}$ \\
\hline & $\begin{array}{l}\text { Softwareinstallation. } \\
\text { Einlesen von Bibliotheken }\end{array}$ & $\begin{array}{l}\text { CD-ROM-System } 600 \mathrm{MB} \text {, } \\
\text { SCSI für HP9000 }\end{array}$ \\
\hline & $\begin{array}{l}\text { Erhöhung der Festplattenkapazität } \\
\text { zum Speichern umfangreicher } \\
\text { Nutzerdaten }\end{array}$ & $\begin{array}{l}\text { Upgrade } 1 \mathrm{~GB} \text { statt } 525 \text { MB-Platte } \\
\text { für HP715xx }\end{array}$ \\
\hline 1.1 & $\begin{array}{l}\text { Ausgabe von Zeichnungen } \\
\text { A3 bis } A 0\end{array}$ & $\begin{array}{l}\text { Plotter HP-Designjet } 600 \mathrm{AO} \\
\text { Ethernet-Netzwerkkarte für HP MO-Bus } \\
\text { Speichererweiterung } 8 \mathrm{MB} \\
\text { Supportpack ( } 3 \text { Jahres-Hardware-Service) }\end{array}$ \\
\hline \multirow[t]{3}{*}{2} & $\begin{array}{l}\text { 3-D CAD/CAM-Arbeitsplatz für } \\
\text { Pro/Engineer }\end{array}$ & $\begin{array}{l}\text { Workstation HP9000/715-75. CRX } \\
32 \text { MB RAM. } 525 \text { MB Festplatte inkl. } \\
\text { UNIX-Betriebssystem }\end{array}$ \\
\hline & Datentransfer per Diskette & Diskettenlaufwerk für HP9000 \\
\hline & $\begin{array}{l}\text { Erhöhung der Festplattenkapazität } \\
\text { zum Speichern umfangreicher } \\
\text { Nutzerdaten }\end{array}$ & $\begin{array}{l}\text { Upgrade } 1 \text { GB statt } 525 \text { MB-Platte } \\
\text { für HP715xx }\end{array}$ \\
\hline 2.1 & Datensicherung & Gigatape-System 4 GB \\
\hline 2.2 & $\begin{array}{l}\text { Drucker für Zeichnungsausgabe } \\
\text { DIN A } 4 \text { und DIN A } 3 \text { (verkleinert) }\end{array}$ & $\begin{array}{l}\text { Laserdrucker HP Laserjet } 4 \mathrm{~L} \text {. } \\
\text { Ethernet-Netzwerkkarte für HP MiO-Bus }\end{array}$ \\
\hline 3 & $\begin{array}{l}\text { PC-Arbeitsplatz für den Geschäfts- } \\
\text { führer }\end{array}$ & $\begin{array}{l}\text { PC AT } 486 / 66 \text { VL.B } 16 \text { MB: } 540 \text { MB HD: } \\
\text { CD-ROM. } 3.5^{\prime \prime} \text { DLW: Netzwerkkarte; } \\
\text { 17"-Monitor }\end{array}$ \\
\hline 3.1 & Drucker für Geschäftskorrespondenz & Laserdrucker HP Laserjet $4 \mathrm{~L}$. \\
\hline 3.2 & $\begin{array}{l}\text { Senden und Empfangen von Fax- } \\
\text { dokumenten }\end{array}$ & Externes Modem ML28800TL. \\
\hline $4=$ & Arbeitsplatz für Schreibkraft & $\begin{array}{l}\text { PC AT } 486666 \text { VLB } 8 \text { MB: } 340 \mathrm{MB} \text { HD: } \\
3.5^{-1} \text { DLW: Netzwerkkarte: } 15^{\circ-} \text {-Monitor }\end{array}$ \\
\hline 4.1 & $\begin{array}{l}\text { Drucker für Geschaftskorrespon- } \\
\text { denz, Ausgabe von Faxdokumenten }\end{array}$ & Laserdrucker HP Laserjet $4 \mathrm{~L}$. \\
\hline 5 & NC-Verwaltung & $\begin{array}{l}\text { PC AT 486/66 VLB } 8 \text { MB; } 540 \mathrm{MB} \text { HD; } \\
\text { 3.5" DLW; Netzwerkkarte; } 15^{\prime \prime} \text { :Monitor }\end{array}$ \\
\hline 5.1 & Protokolldrucker & Nadeldrucker NECP62: EBE \\
\hline 6 & Werkzeugeinstellung & $\begin{array}{l}\text { PC AT 486/66 VLB } 8 \mathrm{MB} ; 540 \mathrm{MB} \text { HD; } \\
\text { 3.5" DtW: Netzwerkkarte: } 15^{*} \text {-Monitor }\end{array}$ \\
\hline 7 & $\begin{array}{l}\text { Meßmaschine } \\
\text { Erfassung und Verarbeitung der } \\
\text { ermittelten Geometriedaten }\end{array}$ & $\begin{array}{l}\text { PC AT } 486666 \text { VLB } 8 \text { MB: } 540 \mathrm{MB} \text { HD: } \\
\text { 3.5" DLW; Netzwerkkarte; } 15^{*} \text {-Monitor }\end{array}$ \\
\hline
\end{tabular}

Tabelle 4: Hardware-Anforderungen zur Konfiguration aus Bild 2 (Vorzugslösung)

\subsection{Zusammenfassung}

Mit diesen ausgewählten Abschnitten aus der Studie zur Einfuihrung eines durchgängigen CAD-CAM-Systems bei einem kleinen Unternehmen für Formenbau wurde die Vorgehensweise mit punktuellen inhaltlichen Aussagen vorgestellt.

Die Technische Fachhochschule Wildau hat damit den ersten Teil einer umfangreichen Transferleistung erbracht, zudem wurden Fördermittelanträge gemeinsam mit dem AU erarbeitet und bei der T.IN.A. Brandenburg eingereicht. Sollte auch diese Etappe in einer erträglichen Zeit mit Erfolg abgeschlossen werden, so kann der Prozeß der Planung und Realisierung fortgesetzt bzw. durchgeführt werden - eine gemeinsame Aktion von auftraggebenden Unternehmen, Systemlieferanten und der Technischen Fachhochschule Wildau.

Ein kleines Unternehmen hat nur durch das konstruktive Zusammenwirken aller Partner die Chance, bei laufender Produktion die Einführung einer durchgängigen CAD-CAM-Lösung ohne Verluste zu realisieren. Die konstruktiven und fertigungstechnischen Erfahrungen des $\mathrm{AU}$, die Erfahrungen im Umgang mit CAD-CAM-Lösungen (hard- und softwareseitig) der TFH Wildau sowie eine komplette Lieferung und Inbetriebnahme durch den Lieferer, gepaart mit der Kombination aus AU-Mitteln und Fördermitteln sind die Voraussetzungen für eine erfolgreiche Systemeinführung.

\section{Rechnerintegrierte Produktion in der Lehre}

Aus den Untersuchungen der letzten Jahre in unterschiedlichsten Unternehmen ergibt sich die Notwendigkeit, in der Fachhochschulausbildung bei angehenden Ingenieuren des Fachbereiches Maschinenbau diesen Sachverhalt praxisnah zu vermitteln.

\subsection{DV-Ausbildung im Fachbereich Maschinenbau}

Die DV-Ausbildung wurde aus dem Anforderungsbild der Praxis, wie anschließend geschildert, gegliedert in

- DV-Grundlagenausbildung,

- PC-Netze,

- Datenbanken,

- Rechnerintegrierter Betrieb.

Die Gliederung stellt gleichzeitig den Namen der entsprechenden Lehrgebiete, die sich vom 2 . Semester bis hin zum 7. Semester spannen, dar.

Die DV-Grundlagenausbildung geht über 2 Semester mit insgesamt 6 Semesterwochenstunden. In diesem Komplex soll ein gewisser Niveauabgleich der Studierenden erfolgen. Es werden Themen behandelt wie

- Einführung (Information, Codierung, Zahlensystem, Bildschirmarbeitsplatz, Datensicherung, Datenschutz),

- Rechnersysteme aus Nutzersicht (Zentraleinheit, Beispielsysteme, Prozessor, Speicher, periphere Geräte, Netze),

- Betriebssysteme und Nutzungstechnologie (Arten, Strukturen, Nutzungstechnologien, Nutzung eines Einplatzbetriebssystems, Programmiersprache),

- Übungskomplexe.

Im Lehrgebiet Informationssysteme/PC-Netze, welches über 1 Semester mit 2 Semesterwochenstunden geht, werden Themen behandelt wie

- Rechnerkommunikation,

- Netzwerke,

- NetWare Grundlagen (Anwenderkurs),

- Konfiguration einer Firma.

Im Lehrgebiet Informationssysteme/Datenbanken, das ebenfalls über 1 Semester in 2 Semesterwochenstunden läuft, werden folgende Themen behandelt:

- Objekte, Datensätze, Beziehungen, Dateien,

- Datenbankorganisation, Zugriffsmethoden,

- Datenbankmodelle,

- Datenbankgestaltung,

- Programmierung einer Datenbank,

- Übungskomplex.

Den Abschluß bildet das Lehrgebiet Rechnerintegrierter Betrieb. Hier wird versucht, den gesamten Prozeß von der Entwicklung bis hin zur Fertigung eines Erzeugnisses auch unter Nutzung von Robotertechnik zu verfolgen. Dies geschieht an unterschiedlichsten Hard- und Softwarebeispielen. Der Studierende erhält die Möglichkeit, an Unternehmensaufgaben zu arbeiten, sich im Team zu bewähren. An Studienaufgaben unterschiedlichster Art kann er sein Problemlösevermögen, Koopera- 
tionsfähigkeit, Lernerfahrung und Kreativität erproben. Zur Vertiefung von speziellen Kenntnissen wird auch ein Wahlpflichtfach zur Thematik „Rechnerintegrierter Betrieb" - mit speziellen Themen - angeboten.

\subsection{Schlußbemerkung}

Qualifikation für einen bestimmten Beruf bedeutet auch den Erwerb einer Menge von Fähigkeiten, Fertigkeiten und Kenntnissen. Nur so kann der Absolvent schnell befähigt werden, seine Beruftstätigkeit auszuüben. In diesem Sinne gedacht, ist das Konzept aus der Praxis entlehnt für die Praxis. Dies trifft besonders für die erfolgreiche Tätigkeit in einem kleinen Unternehmen zu.

\section{Literatur}

[1] Scheer, A.-W.: CIM im Mittelstand. Fachtagung Saarbrücken, Febr. 1991. Springer-Verlag Berlin, Heidelberg.

[2] Hübner: Integration und Informationstechnologie im Unternehmen. München 1979.

[3] Lehmann, H.: siehe in $\mid 4$, S. 768-774

[4] Treuling, W. (Hrsg.): Auswirkungen unterschiedlicher Integrationsgrade des EDV-Einsatzes auf die Zielerreichungsgrade von betrieblichen Nutzungsgrößen. Schlußbericht an die Deutsche Forschungsgemeinschaft. FIR Aachen, 1988.

[5] Sauerbrey, G.: Planung organisatorischer Veränderungen von Integrationsansätzen

[6] Bullinger, H.-J.: Die CIM-fáhige Fabrik. Zukunftssichernde Planung und erfolgreiche Praxisbeispiele. 8. IAO-Arbeitstagung, 4. 5. Mai 1988, Stuttgart.

[7] AWF, Ausschuß für wirtschaftliche Fertigung: Integrierter EDVEinsatz in der Produktion; Begriffe, Definitionen, Funktionszuordnung. Eschborn, 1985.

[8] Köhl, E. u. a.: CIM zwischen Anspruch und Wirklichkeit; Erfahrungen, Trends, Perspektiven. RKW Verlag, Verlag TÜV Rheinland, 1981

[9] Grochla, E.: Handwörterbuch der Organisation. 2. Auflage, Stuttgart, 1980.

|10| Hentschel, B.; Kuchling, K.; Schubert, G.; Bärthel, H.: Konzeptionelle Vorstellungen zur Schaffung eines durchgängigen CADCAM-Systems. Technische Fachhochschule Wildau, Beratungsbericht 11/1994.

[11] Hentschel, B.; Kuchling, K.; Schubert, G.; Bärthel. H.: Konzeptionelle Vorstellungen zur Schaffung eines durchgängigen CADCAM-Systems. Technische Fachhochschule Wildau, Beratungsbericht 03/1995.

[12] Firmenschriften und Angebote zu CAD-CAM-Systemen, Systemmodulen sowie Meßeinheiten und Werkzeugvoreinstellgeräten. so u. a. zu Pro/ENGINEER, CAMAX, ME 10 und PE/SolidDesigner.

\section{Verfasser}

\section{Dr--Ing. Helmut Bärthel}

Technische Fachhochschule Wildau

Technologietransferstelle

Tel. (0 33 75) 507-210

Prof. Dipl.-Ing. Karlheinz Kuchling

Technische Fachhochschule Wildau

Fachbereich Betriebswirtschaft/Wirtschaftsinformatik

Tel. (0 33 75) 507-171

Dipl.-Ing. Günter Schubert

Technische Fachhochschule Wildau

Leiter Hochschulrechenzentrum

Tel. (0 33 75) 507-186 